\subsection{6 doi: 10.15789/2220-7619-2018-4-6.46 MOLECULAR EPIDEMIOLOGY OF TUBERCULOSIS IN KAZAKHSTAN, 2006-2018}

Y. Skiba ${ }^{1,2}$, I. Mokrousov ${ }^{3}$, D. Nabirova ${ }^{4}$, B. Toksanbayeva ${ }^{6}$, E. Maltseva $^{1}$, G. Ismagulova ${ }^{1}$, D. Naizabaeva ${ }^{1}$, A. Bissenbay ${ }^{1}$, N. Yurkevich ${ }^{1}$, V. Bismilda ${ }^{6}$, L. Chingissova ${ }^{6}$, Z. Sapiyeva ${ }^{5}$, S. Ismailov ${ }^{6,7}$, D. Moffett ${ }^{4}$, M. Adenov ${ }^{6}$

${ }^{1}$ Aitkhozhin Institute of Molecular Biology and Biochemistry, Almaty, Kazakhstan; ${ }^{2}$ Almaty Branch of National Center for Biotechnology at Central Reference Laboratory, Almaty, Kazakhstan; ${ }^{3}$ St. Petersburg Pasteur Institute, St. Petersburg, Russia $;{ }^{4}$ Centers for Disease Control and Prevention, Central Asia Regional Office, Almaty, Kazakhstan; ${ }^{5}$ Interdistrict Tuberculosis dispensary, Ministry of Health, Almaty, Kazakhstan; ${ }^{6}$ National Center of Tuberculosis Problems, Ministry of Health, Almaty, Kazakhstan; ${ }^{7}$ The Global Fund, Geneva, Switzerland

The implementation of various projects aiming to develop basis of molecular-epidemiological monitoring of tuberculosis infectious agent by combining scientific and technical potential of specialists from several research and medical centers resulted in extensive experience and contribution to understanding epidemic process of tuberculosis in Kazakhstan. In particular, we established an updated information database of genetic profiles using 24-MIRUVNTR and spoligotyping, a set of reagents designed to carry out molecular profiling of mycobacterial isolates, as well as protocols based on reduced and expanded panels for stream high-throughput screening in 96- and 384-well format.

The studies were conducted on clinical isolates of $M$. tuberculosis, collected from 2006 to 2018 in hospitals of Kazakhstan. The samples were characterized by the resistance to first and second line antimicrobials using cultivation on LowensteinJenssen media and BACTEC MGIT 960. Genotyping: manual 24MIRUVNTR-typing (Supply et al., 2006) and spolygotyping (Kamerbeek et al., 1994), compared with MIRUVNTRplus and SITVIT_WEB databases. Additional typing of hypervariable loci QUB-18, QUB3232, VNTR-3820, VNTR-4120 was used when insufficient genotypes' differentiation was observed (Iwamoto et al., 2007; AllixBeguec et al., 2014). Genetic polymorphism of drug resistance was determined by TB-TEST system (BIOCHIP-IMB, Russia), and GenoType MTBDRplus kits (Hain Lifescience, Germany).

Our pilot study of 2007-2008 established that Beijing strains present a special epidemic threat for Kazakhstan as they are the main reason for the majority of MDR tuberculosis cases and are widely distributed in different regions of Kazakhstan (Skiba et al., 2015). Further study (2012-2014, with 576 genotyped samples of drug-resistant strains) allowed not only to confirm the previous conclusions, but also clarified prevalence and impact of other genetic families in Kazakhstan. Both of these studies led to identification and confirmation of a separate genetic group, which we named KAZ-1.

These studies have shown some cases of tuberculosis caused by several mycobacterial strains simultaneously. We have also encountered genotype changes in patients during their hospital treatment. This finding formed the basis for the next project on the study of nosocomial transmission of drug-resistant tuberculosis (2015-2017). The result of this project was registration of several cases of genotype change in hospitalized patients at different treatment stages. This targeted monitoring also helped to record the first case of LAM RD-Rio strain in Kazakhstan.

The overall results inspired us to use the developed research algorithm that combines reduced VNTR panel with TB-TEST biochip system in the ongoing research to reveal the connection between drug-resistance mutations and strain genotype, and to better understand the epidemic process of tuberculosis in Kazakhstan.
6.47

doi: 10.15789/2220-7619-2018-4-6.47

\section{NEXT-GENERATION SEQUENCING OF DRUG RESISTANT MYCOBACTERIUM TUBERCULOSIS STRAINS - FIRST SLOVENIAN EXPERIENCE}

E. Sodja ${ }^{1}$, N. Toplak ${ }^{2}$, S. Koren ${ }^{2}$, M. Kovač², S. Truden ${ }^{1}$, M. Žolnir-Dovč ${ }^{1}$

${ }^{1}$ University Clinic of Respiratory and Allergic Diseases Golnik, Golnik, Slovenia; ${ }^{2}$ Omega d.o.o., Ljubljana, Slovenia

Slovenia is low-incidence country with incidence rates of tuberculosis (TB) cases around 6.0 in last several years. In our country, the percentage of resistant $\mathrm{TB}$ is very low with sporadic cases of multidrug resistant (MDR) TB. The aim of our study was to examine the feasibility of a fulllength gene analysis for the drug resistance related genes (inh $A, k a t G, r p o B, e m b B$ ) using Next-generation sequencing Ion Torrent technology and compare the results with those obtained from conventional phenotypic drug susceptibility testing (DST) in 61 TB isolates from our National mycobacterial culture collection. TB strains included were either susceptible or mono-, poly-, or multidrug resistant by phenotypic DST. High concordance between genetic (Ion Torrent technology) and standard phenotypic DST testing for isoniazid, rifampicin and ethambutol was observed with sensitivities of $68.2 ; 100$ and $100 \%$, and specificities of 100 ; 80 and $88.2 \%$, respectively. In conclusion, the next-generation sequencing analysis successfully predicted drug resistance with significant shortening of time needed to obtain the resistance profiles from several weeks to just a few days.

\section{WGS IN ROUTINE DIAGNOSTICS OF TUBERCULOSIS -} PREDICTION OF DRUG RESISTANCE \section{AND GENOTYPING}

H. Soini

National Institute for Health and Welfare, Department of Health Security, Helsinki, Finland

The aim of the study was to compare the performance of whole genome sequencing (WGS) and conventional assays for drug susceptibility testing and genotyping of Mycobacterium tuberculosis in a routine laboratory.

All M. tuberculosis cultures sent to the Finnish mycobacterial reference laboratory in 2014 were tested by Mycobacteria Growth Indicator Tube (MGIT) for firstline drug susceptibilities. Genotyping was performed by 24 loci MIRU-VNTR typing and spoligotyping. WGS was performed with the Illumina MiSeq system. For prediction of drug susceptibility, the data were analyzed using five software tools (PhyResSE, Mykrobe Predictor, TB Profiler, TGS-TB and KvarQ). Clustering analysis was performed using Ridom SeqSphere+ (Ridom GmbH, Germany) cgMLST v2 (2891 targets) for genomes assembled by Burrows-Wheeler Aligner (bwa). Isolates with allelic distance $\leq 12$ formed a cgMLST cluster. In addition, SNP analysis using the GATK tools (Broad institute, Cambridge, MA, USA) was performed and clusters based on distance of $\leq 12$ and $\leq 1$ SNPs were formed.

The sensitivity of the five software tools to predict any resistance among strains was almost identical, ranging from $74 \%$ to $80 \%$, and specificity was more than $95 \%$ for all software tools except for TGS-TB. The sensitivity and specificity to predict resistance to individual drugs varied considerably among the software tools.

Among the 211 isolates, 15 clusters comprising 36 isolates $(19.9 \%)$ were found by conventional genotyping. Of these, 15 isolates $(36.1 \%)$ were clustered similarly to six clusters also by cgMLST analysis. Furthermore, four strains that did not cluster by conventional genotyp- 
ing clustered by cgMLST analysis resulting in 19 (9.0\%) clustered isolates by cgMLST. By clustering analysis with the distance $\leq 12$ SNPs, 18 isolates clustered into 7 clusters. With the 1 SNP cut-off, three clusters with a total of seven strains were found and these were similarly clustered also by cgMLST and conventional genotyping analysis.

A reliable prediction of drug susceptibility can be obtained with WGS combined with data analysis with software tools. In routine practice, $M$. tuberculosis isolates can be screened with WGS for mutations associated with drug resistance, and only resistant strains confirmed with the MGIT system. Compared to conventional genotyping methods, WGS analysis is more discriminatory, reducing the risk of false clustering and unnecessary contact tracing.

\subsection{9 doi: 10.15789/2220-7619-2018-4-6.49 \\ SINGLE NUCLEOTIDE POLYMORPHISMS IN hsp65 AND MACPPE12 GENES OF MYCOBACTERIUM AVIUM subsp. HOMINISSUIS}

D. Starkova ${ }^{1}$, T. Iwamoto ${ }^{2}$, A. Vyazovaya ${ }^{1}$, V. Molchanov ${ }^{3}$, V. Zhuravlev ${ }^{4}$, B. Vishnevsky ${ }^{4}$, O. Narvskaya ${ }^{1,4}$

${ }^{1}$ St. Petersburg Pasteur Institute, St. Petersburg, Russia; ${ }^{2}$ Department of Infectious Diseases, Kobe Institute of Health, Kobe, Japan; ${ }^{3}$ St. Petersburg State Chemical Pharmaceutical University, St. Petersburg, Russia; ${ }^{4}$ St. Petersburg Research Institute of Phthisiopulmonology, St. Petersburg, Russia

Mycobacterium avium subsp. hominissuis (MAH) represents a group of environmental bacteria known as opportunistic pathogens of animals and humans, especially HIV positive. Polymorphisms in hsp65 and MACPPE12 genes are used for identification, intra-subspecies differentiation and phylogenetic studies of $M A H$ populations.

The aim of our study was to identify single-nucleotide polymorphisms (SNPs) in hsp65 and MACPPE12 genes of clinical isolates from Russian patients with pulmonary and disseminated mycobacteriosis and to assess phylogenetic relationships of geographically distant $M A H$ populations.

The sequence analysis of the 3'-portion of the hsp65 gene and MACPPE12gene was applied for $40 \mathrm{MAH}$ strains isolated from humans with mycobacteriosis (including 19HIV-positive) in St. Petersburg, Russia (2008-2011). The nucleotide sequences were aligned to the reference genome of M. avium subsp. hominissuis 104 (NC_008595.1.).

In total, the $40 \mathrm{MAH}$ strains were classified into three different hsp65 sequevars: code 1 , code 2 and code 3 . The majority of $M A H$ strains $(72.5 \%)$ belonged to code 1 , the same sequevar as for $M A H$ strain 104 . The code 2 and code 3 included $3(7.5 \%)$ and $8(20 \%)$ strains, respectively. The largest $h s p 65$ sequevar code 1 has observed only in $4.7 \%$ of isolates from Japan and absent in Korean human isolates. The sequevars code 1 and code 2 predominated among $M A H$ strains in the USA, Canada, Belgium.

The sequence analysis of the MACPPE12 gene revealed 20 SNPs grouped into nine sequevars at the nucleic acid level: NA01, NA02, NA03, NA06, NA10, NA13, NA14, NA19, and NA_Rus01. Among 20 SNPs eight were nonsynonymous resulting in seven sequevars at the amino acid level: AA01, AA02, AA04, AA07, AA08, AA13, and AA Rus01. The sequevar AA02 consisted of three different NA variants with synonymous SNPs profiles: NA02, NA03, and NA06. Half of the $M A H$ strains belonged to the sequevar AA02 (type NA02). The predominant cluster AA02 (type NA02)/code 1 and the unique variant AA Rus01 (NA Rus01) were identified among $M A H$ strains from Russia. The present study demonstrated the prevalence of the sequevar AA02 in MAH strains isolated from humans in Russia, Japan, and Korea.
Thus, we confirmed the relative conservativeness of the nucleotide sequence of the hsp 65 gene but the polymorphism of the MACPPE12 gene. A comparative analysis of the SNPs profiles of the hsp 65 and MACPPE12 genes allowed to identify differences and similarities between geographically distant populations of $M A H$, which highlighted the variability of the global population of M. avium species.

\section{MOLECULAR EPIDEMIOLOGY OF TUBERCULOSIS} IN ALBANIA (2006-2011)

S. Tafaj ${ }^{1}$, P. Kapisyzi ${ }^{1}$, E. Borroni ${ }^{2}$, A. Trovato ${ }^{2}$, D. Bardhi ${ }^{1}$, H. Hafizi ${ }^{1}$, S. Bala ${ }^{1}$, D. Cirillo ${ }^{2}$

${ }^{1}$ National TB Reference Laboratory, University Hospital Shefqet Ndroqi, Tirana, Albania:

${ }^{2}$ Emerging Bacterial Pathogens Unit, Division of Immunology and Infectious Diseases, IRCCS San Raffaele Scientific Institute, Milan, Italy

Tuberculosis (TB) epidemics in Albania has been stable over the past years with a gradual decreasing incidence (from 18.7 to 14.8 per 100000 inhabitants, in the period 2001-2016) with a slight deterioration in 2013 (16.8 per 100000 inhabitants). First insight data (2008) on TB molecular epidemiology showed a moderate Recent Transmission Index (RTI) (28\%) and a high level of genetic diversity.

We aimed with this study to better understand the correlation of ubiquitous and autochthonous Mycobacterium tuberculosis complex (MTBC) genotypes with available demographic and epidemiologic data over a six-year period, in Albania.

MTB strains isolated in Albania ( $\mathrm{n}=745,1$ isolate per patient) between 2006 and 2011 were analyzed by spoligotyping and MIRU-VNTR typing by 24 loci scheme. The data obtained were compared with MIRU-VNTRplus database. Using molecular typing $486(65.23 \%)$ isolates (patients) were distributed into 113 clusters and the remaining 259 (34.77\%) isolates had a unique pattern. The cluster sizes ranged from 2 to 21 isolates per cluster. RTI $((\mathrm{nc}-\mathrm{c}) / \mathrm{n})$ resulted $50.07 \%$. The most predominant lineages were Ghana $(28.59 \%)$, Haarlem (19.73\%) UgandaI (18.79\%), LAM (7.11\%), Ural $(5.64 \%)$, TUR $(3.89 \%)$ and Caprae (3.49\%). Other lineages identified were Cameroon $(1.74 \%), \mathrm{X}(1.48 \%)$, S $(1.21 \%)$, Bovis $(0.54 \%)$, Delhi/CAS $(0.54 \%$, Beijing $(0.4 \%)$ and West African $2(0.13 \%)$. This study highlighted the predominance of five shared spoligotypes: ST 53 (T1) $(\mathrm{n}=166,22.28 \%)$, ST 4 (LAM3 and S/convergent) $(\mathrm{n}=39,5.23 \%)$ and ST $42($ LAM9) $(\mathrm{n}=38,5.10 \%)$ ST 613(T1) $(n=37,4.97 \%)$ and ST $47(\mathrm{H} 1)(\mathrm{n}=35,4.70 \%)$. Of the unknown spoligotype signatures three were more frequent than others $(4.70 \%, 3.22 \%, 3.22 \%)$, their origin and historical link to other genotypes is yet unknown. Among the MLVA MtbC15-9 types, MLVA 15411-85 and MLVA 15419-69 (both of unknown spoligotype signatures) resulted the predominant types involved in recent transmission in Albania (two biggest clusters identified with 21 and 19 identical isolates respectively).

In conclusion, MTBC genetic population in Albania is highly heterogenous. TB epidemics in Albania is fueled mostly by evolutionary-recent lineages. It is largely dedicated to recent transmission (50.07\%). Autochthonous genotypes result linked to the 2 biggest clusters identified. One of them is found exclusively in Tirana (MLVA 15411$85)$. The new MTBC genotypes will require further molecular characterization. 